\begin{tabular}{|c|c|c|c|c|c|}
\hline MUNIBE Antropologia-Arkeologia & $n^{\circ} 67$ & $325-337$ & DONOSTIA & 2016 & ISSN 1132-2217 • eISSN 2172-4555 \\
\hline
\end{tabular}

\title{
Del medio natural a los paisajes pastorales. Ocupación de las zonas de alta montaña en los Pirineos centrales de Cataluña desde el Mesolítico a la Edad del Bronce (c. 9000-1000 cal ANE)
}

\author{
From natural environments to pastoral landscapes. Human occupations \\ in the high mountains areas of the central Pyrenees of Catalonia, \\ since the Mesolithic to Bronze Age (ca. 9.000-1.000 cal BC)
}

PALABRAS CLAVES: Pirineos, Neolítico, arqueología del paisaje, ecología social, pastoralismo.

GAKO-HITZAK: Pirinioak, Neolitoa, paisaiaren arkeologia, ekologia soziala, artzantza.

KEY WORDS: Pyrenees, Neolithic, environmental archaeology, socio-ecology, Pastoralism.

David RODRÍGUEZ ANTÓN(1), Ermengol GASSIOT BALBÈ (1), Niccoló MAZZUCCO(2),
Ignacio CLEMENTE CONTE(2), Laura OBEA GÓMEZ(1) y David GARCIA CASAS

\section{RESUMEN}

En este artículo presentamos los resultados de los trabajos de investigación arqueológica y paleoambiental llevados a cabo entre 2000 y 2014 en el Parque Nacional de Aigüestortes i Estany de Sant Maurici (PNAESM) y en su área periférica. Dichos trabajos comprenden la prospección sistemática de la mayor parte del territorio, entre 1500 y 3000 m de altitud, que ha permitido documentar 350 sitios arqueológicos, proporcionando 78 dataciones radiocarbónicas que comprenden una secuencia de ocupación de la zona de 10700 años. Asimismo, se ha completado la excavación de tres yacimientos arqueológicos: Cova del Sardo de Boí, Abric de l'Estany de la Coveta I y el Dolmen de la Font dels Coms. El análisis arqueológico y paleoambiental de los sitios, de diversos perfiles y de 5 registros lacustres permiten modelizar la historia de las actividades económicas humanas en la alta montaña y el proceso de construcción de los primeros paisajes pastorales.

\begin{abstract}
LABURPENA
Artikulu honetan, Aigüestortes i Estany de Sant Maurici (PNAESM) parke nazionalean eta parkearen eremu periferikoan 2000. urtetik 2014ra eginiko ikerketa arkeologiko eta paleoanbientalen emaitzak aurkeztuko ditugu. Eginiko lanen artean daude lurraldearen zatirik handienaren prospekzio sistematikoa, 1.500-3000 m-ko altitudean egina, eta horri esker, 350 gune arkeologiko dokumentatu eta 78 datazio erradiokarboniko eskuratu ditugu, gunean 10700 urtean izandako okupazioaren sekuentziari lotutakoak. Era berean, hiru aztarnategi arkeologikoren indusketa ere burutu dugu: Cova del Sardo de Boí, Abric de l'Estany de la Coveta I eta Dolmen de la Font dels Coms. Guneen, profilen eta 5 aintzari-erregistroren inguruan egindako azterketa arkeologiko eta paleoanbientalak aukera ematen dute goi mendietako giza jarduera ekonomikoen historia eta lehen artzantza-paisaien eraikitze-prozesua modelizatzeko.
\end{abstract}

\begin{abstract}
This paper presents the results of the archaeological and palaeoecological research conducted between 2000 and 2014 in the Aigüestortes i Estany de Sant Maurici National Park (PNAESM). This included the systematic survey of the most of the National Park territory, included at altitudes between 1500 and $3000 \mathrm{~m}$ a.s.l. During the surveys, 350 archaeological sites have been detected and 78 radiocarbon dates have been realized, providing a sequence of human occupation from $10700 \mathrm{cal}$ BP to modern times. In addition, three archaeological sites with stratified deposits have been fully excavated: Cova del Sardo de Boí, Abric de l'Estany de la Coveta I and Dolmen de la Font dels Coms. In parallel, 5 sedimentary cores have been extracted from lakes and one pigbog, from the Natural Park of Alt Pirineu (PNAP) and National Park (PNAESM) areas, providing a full palaeoecological sequence from the last 17.000 years cal BP. This archaeological and palaeoecological dataset (e.g. soil-sites and lake records) allowed advancing new models about the anthropization of the high-altitude areas and the social construction of pastoral landscapes.

At the current state of research, the first signs of an anthropic input on the mountain landscape in the Pyrenees date back to ca. $5000 \mathrm{cal}$ $\mathrm{BC}$. The last 15 years of survey and research in the area have demonstrated that first human presence is mainly related to the development of an agro-pastoral economic system, also known as 'Neolithic'. In this context, mid- and high-altitude areas do not appear to be isolated spaces. In contrast, the latest archaeological data suggests a strong connection between mountains, plains and coastal areas of the NE of the Iberian
\end{abstract}

\footnotetext{
(1) Universitat Autònoma de Barcelona UAB. Departament de Prehistòria. Edifici B. 08193. Bellaterra (Cerdanyola del Valles), Barcelona. Correos electrónicos: david.anton79@gmail.com; ermengol.gassiot@uab.cat; David.garc83@gmail.com; laura.obea87@gmail.com

(2) CSIC - Institución Milá y Fontanals (IMF), Departamento de Arqueología y Antropología. C/ Egipciaques, 15. 08001 Barcelona. Correos electrónicos: ignacio@imf.csic.es; nicco.mazzucco@gmail.com
} 
Peninsula. This area is transitional between the relatively arid inland plains and the alpine landscapes. Here, during a period of 3000 yrs, the palaeoecological and archaeological data shows an increase of anthropic pressure. In this paper we discuss the results of a multidisciplinary research project carried out in the Sant Nicolau valley, a glacial valley located in the western Catalan Pyrenees at altitudes between 1500 to $3000 \mathrm{~m}$ a.s.l., and in the rest of the Nacional Park (PNAESM). Our research involves several complementary approaches and disciplines: 1) an extensive survey of the area and the integration of all types of archaeological evidences on a regional GIS; 2) a diachronic study of all the excavated sites, which includes an economic approximation of the archaeological materials and integrated archaeobotanical analyses; 3) an analysis of the landscape evolution through palaeosols and lacustrine sedimentary cores.

The first results of this integrated approach are encouraging, showing dynamics in the occupation of the mountains that otherwise would be impossible to detect. In the Nacional Park area human presence seems to be sporadic until the first half of the $\mathrm{V}$ millennium cal BC. The first signs of anthropogenic fires, dated between 5200 and 4940 cal BC, seem to anticipate of only a few hundred years the appearance of a major archaeological record at Sant Nicolau button valley. A clear human occupation is dated between 4802 and 4368 cal AC at Cova del Sardo site, and is mainly associated with the exploitation of the subalpine stage for pastoral purposes by groups of southern provenance. Successively, the human presence becomes more discontinuous between 4229 and 3375 cal AC. Gradually moves toward higher altitudes. Indeed an increasing number of sites are established above 2000 m a.s.l., between 3484 and 2345 cal AC. The analysis of the archaeological artefacts suggests that the catchment area of these Neolithic pastoral groups goes from the Ebro Basin to the alpine areas of the Axial Pyrenees. We suggest that the modern landscape, far from being a 'natural' environment, is the result of a long-term process of anthropic transformation, starting VII millennia ago.

\section{1.- INTRODUCCIÓN}

La aproximación tradicional a las zonas de alta montaña, durante el pasado siglo XX, se había enfocado siempre desde un punto de vista conservacionista, en tanto se veían éstas como territorios vírgenes, ignotos, naturales o escasamente alterados por la presencia humana. De esta visión nace la necesidad social de su protección y conservación, como reservas de un mundo natural primigenio. El primero de estos territorios especialmente protegido por leyes estatales es el Parque Nacional de Yellowstone, en los EUA, creado en 1872. En 1955 se crea con idéntica filosofía el Parque Nacional de Aigüestortes i l'Estany de Sant Maurici (apartir de ahora PNAESM), ubicado entre las cabeceras de las Nogueras Pallaresa y Ribagorzana y de la Garona, en la región central de la cordillera pirenaica, al norte de la provincia de Lleida (Catalunya).

El desarrollo de un nuevo enfoque, más social, en el presente siglo, comienza a prestar interés en estas zonas protegidas ya no sólo al patrimonio natural cuanto al patrimonio cultural (ESTÉBAN et al. 2003). Los nuevos programas de investigación interdiciplinares (CATALAN et al. 2013) demuestran que, en realidad, la construcción del paisaje actual es tanto la consecuencia de la morfología glacial de la cordillera (BORDONAU 1992) y de los cambios climáticos acaecidos en los últimos 17 mil años (PÈLACHS et al. 2011 y 2012), como de la intervención humana ya desde inicios del actual periodo posglacial (PÉREZ-DíAZ et al. 2015). La puesta en marcha de una serie de programas de investigación tanto desde la paleogeografía (SORIANO et al. 2003) como desde la arqueología (GASSIOT et al. 2014) en la región pallaresa durante la primera década del siglo XXI ha permitido poner en valor la existencia de un rico patrimonio cultural, que incluye, desde vestigios de las actividades metalúrgicas de época romana (AUGÉ et al. 2012) hasta la presencia de asentamientos prehistóricos de diversa entidad y cronología (GASSIOT et al. 2010). Esta serie de evidencias de larga duración, tanto arqueológicas, de presencia en el territorio, como paleoambientales, de impacto en el medio, están permitiendo establecer nuevas hipótesis en el proceso de formación de los actuales pai- sajes alpinos (CARNELLI 2002, GALOP 2006, CUNILL et al. 2012, PÉREZ-DÍAZ et al. 2015). Así como explicar los cambios ecológicos acaecidos en el medio durante los últimos 17000 años, no sólo en la medida de los cambios climáticos, sino también como resultado de las diversas actividades económicas humanas, que incluyen desde la ganadería a la agricultura, pasando por la metalurgia, la producción de carbón o las actividades cinegéticas.

\section{2.- ÁREA DE ESTUDIO Y ENFOQUE DE LA IN- VESTIGACIÓN}

\section{1. Área de Estudio}

La zona de estudio a la que hace mención este trabajo se concreta en el PNAESM y su área periférica. Se trata de un territorio de poco menos de $40.000 \mathrm{Ha}$. de extensión y que se ubica en la región central del Pirineo axial a una altitud que se sitúa aproximadamente entre los 1500 y los 3000 m. Geomorfológicamente la geografía del terreno está dominada por el afloramiento del sector oriental del batolito granítico de Posets-Maladeta, siendo el sustrato granítico el dominante, aunque también encontramos afloramientos de esquistos y cuarcitas (DOMINGO et al. 2000). Se trata de un paisaje abrupto formado en la orogenia alpina y con un marcado modelado glaciar reciente. El parque y su entorno se sitúan en la divisoria de aguas de la cordillera, quedando la cabecera de la cuenca de la Garona al norte, en el Valle de Aran, y las cuencas de las nogueras Pallaresa y Ribagorzana, al sur, en los Valles de Sant Nicolau, Vall Fosca y Espot. La geología granítica de la región favorece la abundancia de lagos, unos 272 permanentes, sólo en el PNAESM, además de centenares de pequeños depósitos estacionales, asociados al ciclo de hielo-deshielo anual. Éstos se sitúan desde los circos glaciales hasta los fondos de valle (BORDONAU 1992).

La climatología de la zona está marcada por la altitud, desarrollándose un clima de montaña, relativamente húmedo (entre 1800 y 1000 mm/año) y marcadamente estacional, donde las precipitaciones en forma sólida son las más abundantes, acumulándose una extensa cobertura nival en invierno y primavera, que se 
deshiela casi en su totalidad en verano-otoño, salvo en algún pequeño glaciar marginal de tipo rocoso, en rápido retroceso. Esta climatología favorece el desarrollo de tres pisos bioclimáticos altitudinales en la zona (NINOT y FERRÉ, 2008). Un piso montano, que alcanza los 1600-1800 m, situado únicamente en algunos fondos de valle, y que se caracteriza por una vegetación de tipo boscoso, mixto, generalmente con dominancia de caducifolias: roble (Quercus humilis), abedul (Betula pendula), haya (Fagus sylvatica), fresno (Fraxinus excelsior) y avellano (Corylus avellana), entre otras (Prunus, Alnus, Tilia, Salix, Fraxinus). En zonas de umbría, sobre todo en cara norte, estos bosques de caducifolias se combinan con abetos (Abies alba) y pinos (Pinus sylvestris), que son más dominantes a mayor altitud, ya en la zona inferior del piso subalpino, entre 1800 y 2000 m. Entre los 2000 y los $2300 \mathrm{~m}$ la especie dominante en las zonas forestadas es el pino negro o pino de montaña (Pinus uncinata), que puede combinarse con algunas caducifolias: avellanos (Corylus avellana) y abedules (Betula pendula) en solanas y servales (Sorbus aucuparia) en umbrías; así como con un extenso substrato arbustivo, formado por azaleas (Rhododendron ferrugineum), y arándanos (Vaccinium myrtillus), en umbrías, y enebros (Juniperus communis) y piornos serranos (Cytisus oromediterraneus), en solanas. Entre los 2300 y los 2700, allí donde el substrato permite la formación de suelos, se desarrollan praderas alpinas, muchas veces con presencia de arbustos y algunos pinos aislados. Están formadas por numerosas especies de herbáceas, flores, y juncáceas, y dominadas por los géneros Festuca, Artemisia y Nardus en zonas de pradera seca y en zonas más húmedas por Carex, Luzula y Juncus, principalmente (CAÑELLAS-BOLTÀ et al. 2009).

\subsection{Métodos y materiales}

Desde una perspectiva que buscaba integrar el estudio de la evolución del clima y su impacto en medios particularmente sensibles a los cambios, como las regiones de alta montaña europeas, se comienza a desarrollar ya en el último cuarto del siglo XX una serie de programas de investigación orientados al análisis detallado de los cambios climáticos y la configuración del paisaje de las zonas de alta montaña desde el último ciclo glacial (BORDONAU 1992, MONSERRAT 1992). Ya a inicios del presente siglo, éstos trabajo comienzan a apuntar a un enfoque necesariamente interdisciplinar, que progresivamente irá integrando el impacto humano como un elemento imprescindible en la comprensión de los procesos de cambio y construcción del paisaje pirenaico (SORIANO et al. 2003, PÈLACHS et al. 2007, GASSIOT et al. 2012, CATALAN et al. 2013).

Justo con el nuevo siglo, en el año 2001, lo que con el tiempo acabará siendo el Grup d'Arqueologia d'Alta Muntanya (GAAM) inicia un programa de investigación arqueológica en las zonas altas del Pallars Sobirà que, desde el 2004, se centra en el PNAESM. Comprende entre sus objetivos la prospección sistemática de la práctica totalidad del terreno accesible y la documentación detallada de todas las estructuras arqueológicas halladas y su adscripción cronocultural, con la finalidad de estudiar el proceso de poblamiento de las zonas de alta montaña y la gestión del medio. Para ello ha sido una necesidad constante poder situar en el tiempo las evidencias arqueológicas. Por esta razón, se ha efectuado un programa de datación radiocarbónica de los contextos documentados en excavaciones en extensión y sondeos, que permite caracterizar la secuencia de ocupación de los sitios (GASSIOT et al. 2016).

Durante estos años, se han realizado prospecciones en la mayor parte del territorio accesible a pie del parque que han permitido documentar, en el PNAESM y su zona periférica, 350 sitios arqueológicos (Figura 1). Además, se ha completado la excavación en extensión y análisis arqueológico de tres yacimientos prehistóricos: la Cova del Sardo (Boí, Vall de Boí), el Abric de l'Estany de la Coveta I (Espot) y el Dolmen de la Font desl Coms (Baiasca, Llavorsí) (GASSIOT et al. 2010 y GASSIOT et al. 2014).

Mediante un extenso programa de muestreo y datación de las ocupaciones relacionadas con estos yacimientos se han obtenido 78 dataciones radiocarbónicas, correspondientes a 55 sitios y a un total de 62 fases de ocupación (Tabla 1). Representan una secuencia de ocupaciones que cubre aproximadamente los últimos 10700 años, con algunas discontinuidades relevantes que serán discutidas en este trabajo.

De forma paralela o integrada en proyectos conjuntos, desde 2006 hasta la actualidad, se ha desarrollado una línea de trabajo basada en la paleogeografía (SORIANO et al. 2003) y en el análisis de los cambios climáticos (CATALAN et al. 2001, PÈLACHS et al. 2009). Estos enfoques paleoambientales se han basado principalmente en el estudio de depósitos sedimentarios de diversos tipos: coluvionales (BAL et al. 2010, CUNILL et al. 2012), de turberas (PÈREZ-OBIOL et al. 2012) y, principalmente, lacustres (PÈLACHS et al. 2011 y 2012). Se han utilizado análisis multivariables de elementos susceptibles de definir los cambios ambientales a diferente escala temporal y geográfica, como las diatomeas y algas crisofíceas (PLA y CATALAN 2005), el análisis polínico (CATALAN et al. 2001, CAÑELLAS-BOLTÀ et al. 2009), la sedimentación de la materia orgánica (PÈLACHS et al. 2011 y 2012) o el estudio pedoantracológico (CUNILL et al. 2012). La reconstrucción a escala regional y altitudinal de los cambios climáticos y la evolución de los paisajes del pirineo axial central se ha llevado a cabo mediante la combinación de diversos estudios de archivos sedimentarios, de los que cabe destacar como referencia para la zona de estudio las siguientes columnas sedimentarias que cubren la totalidad del periodo holoceno aquí discutido (Figura 1); de este a oeste: 1) Estany de la Coma de Burg, Vallferrera, situado en la zona sur del Parc Natural del Alt Pirineu (PNAP), a 1821 m s.n.m. (PÈLACHS et al. 2007, 2011 y 2012); 2) Estany 


\begin{tabular}{|c|c|c|c|c|c|c|}
\hline Yacimiento / Sitio & Fase & Código Lab & Datación BP & $\begin{array}{l}\text { Datación } 2 \Sigma \\
\text { cal ANE }\end{array}$ & Material & Contexto \\
\hline Dolmen Font dels Coms & A.5 & KIA 23142 & $9375+/-35$ & $8746-8563$ & Carbón & Agujero de Poste \\
\hline Abric de l'Estany de la Coveta I & N.5 & KIA 29818 & $7845+/-45$ & $7001-6574$ & Carbón & Hogar \\
\hline Forcall de Sant Esperit AS-2 & N.5 & BETA 358572 & $6100+/-30$ & $5200-4940$ & Carbón & Paleosuelo \\
\hline Cova del Sardo de Boí & N.9 & KIA 37689 & $6525+/-45$ & $5609-5376$ & Carbón & Hogar \\
\hline Cova del Sardo de Boí & N.8 & KIA 37690 & $5850+/-40$ & $4802-4602$ & Carbón & Hogar \\
\hline Cova del Sardo de Boí & N.8 & KIA 40878 & $5715+/-35$ & $4681-4462$ & Carbón & Área de combustión exterior \\
\hline Cova del Sardo de Boí & N.8 & KIA 36935 & $5695+/-35$ & $4618-4454$ & Carbón & Área de combustión exterior \\
\hline Cova del Sardo de Boí & N.8 & KIA 40817 & $6586+/-35$ & $4617-4450$ & Carbón & Hogar \\
\hline Cova del Sardo de Boí & N.8 & KIA 41134 & $5645+/-25$ & $4543-4375$ & Carbón & Nivel de ocupación \\
\hline Cova del Sardo de Boí & N.8 & KIA 40815 & $5635+/-35$ & $4540-4368$ & Carbón & Área de combustión exterior \\
\hline Cova del Sardo de Boí & N.7 & KIA 32340 & $5245+/-40$ & $4229-3971$ & Carbón & Nivel de ocupación \\
\hline Cova del Sardo de Boí & N.7 & KIA 26248 & $5060+/-40$ & $3962-3766$ & Carbón & Nivel de ocupación (sondeo) \\
\hline Cova del Sardo de Boí & N.7 & KIA 40816 & $5000+/-30$ & $3939-3702$ & Carbón & Relleno de cubeta \\
\hline Cova del Sardo de Boí & N.7 & KIA 32342 & $4945+/-35$ & $3791-3652$ & Carbón & Hogar \\
\hline Cova del Sardo de Boí & N.7 & KIA 36934 & $4765+/-40$ & $3641-3381$ & Carbón & Nivel de ocupación \\
\hline Cova del Sardo de Boí & N.7 & KIA 37691 & $4715+/-35$ & $3632-3375$ & Carbón & Nivel de ocupación \\
\hline Cova del Sardo de Boí & N.6 & KIA 32351 & $4555+/-30$ & $3484-3104$ & Carbón & Material constructivo \\
\hline Cova del Sardo de Boí & N.6 & KIA 29816 & $4475+/-30$ & $3309-3028$ & Carbón & Hogar \\
\hline Cova del Sardo de Boíll & N.6 & KIA 40850 & $4465+/-30$ & $3308-3024$ & Carbón & Nivel de ocupación \\
\hline Portarró & N.6 & KIA 28276 & $4255+/-40$ & $3007-2696$ & Carbón & Nivel de ocupación (sondeo) \\
\hline Despoblat deth Tuc del Redon & & BETA 377578 & $4420+/-30$ & $3305-2925$ & Carbón & Nivel de ocupación (sondeo) \\
\hline Cova del Sardo de Boí & N.5 & KIA 26251 & $4210+/-35$ & $2901-2677$ & Carbón & Área de frecuentación \\
\hline Cabana de Coma d’Espòs & N.4 & KIA 36936 & $4180+/-30$ & $2886-2667$ & Carbón & Material constructivo (sondeo) \\
\hline Obagues de Ratera & N.3b & KIA 28280 & $4160+/-35$ & $2880-2627$ & Carbón & Nivel de ocupación (sondeo) \\
\hline Cova del Sardo de Boí & N.5 & KIA 32348 & $4090+/-35$ & 2864-2495 & Carbón & Hogar \\
\hline Lac Major de Saboredo II & N.5 & BETA 290113 & $4010+/-40$ & $2832-2462$ & Carbón & Nivel de ocupación (sondeo) \\
\hline Covetes & T.5 & KIA 32341 & $3960+/-30$ & $2571-2347$ & Carbón & Nivel de ocupación (sondeo) \\
\hline Cova de Sarradé & N.4 & KIA 32335 & $3945+-/ 25$ & $2566-2345$ & Carbón & Nivel de ocupación (sondeo) \\
\hline Abric de l'Estany de Xemeneia & T.8 & BETA 278789 & $3290+/-40$ & $1682-1464$ & Carbón & Nivel de ocupación (sondeo) \\
\hline Despoblat i pletiu de la Cova & & BETA 323403 & $2980+/-30$ & $1310-1120$ & Carbón & Nivel de ocupación \\
\hline
\end{tabular}

Tabla 1: Recopilación de todas las dataciones prehistóricas del PNAESM y su área colindante mencionadas en el texto. Las calibraciones han sido realizadas a partir de la curva INCAL 09 / Collecting of all prehistoric sites dating from PNAESM and its adjacent area mentioned in the text. Calibrations were carried out from INCAL 09 curve.

de Estanilles, ubicado al norte del PNAP, a 2245 m s.n.m., en un depósito lacustre de turbera (PĖREZ-OBIOL et al. 2012, CUNILL et al. 2012); 3) Estany Redó de Sant Nicolau, ubicado en la zona central del PNAESM, a $2105 \mathrm{~m}$ s.n.m. (CATALAN et al. 2001); 4) Estany de Llebreta, ubicado en el mismo Valle de Sant Nicolau, a 1618 m. s.n.m. (CATALÁN et al. 2013) y 5) Lac Redon, a la cabecera de la Noguera Ribagorzana, a 2226 m s.n.m., al oeste del PNAESM (PLA y CATALAN 2005).
Finalmente, ambos enfoques, el ambiental y el social, ha acabado convergiendo en el desarrollo de modelos explicativos interrelacionales, que explican la evolución y construcción del paisaje como consecuencia de la interacción entre el clima, la evolución de las formaciones vegetales y las dinámicas de la ocupación y gestión humana del medio (PÈLACHS et al. 2007, CATALAN et al. 2013). 


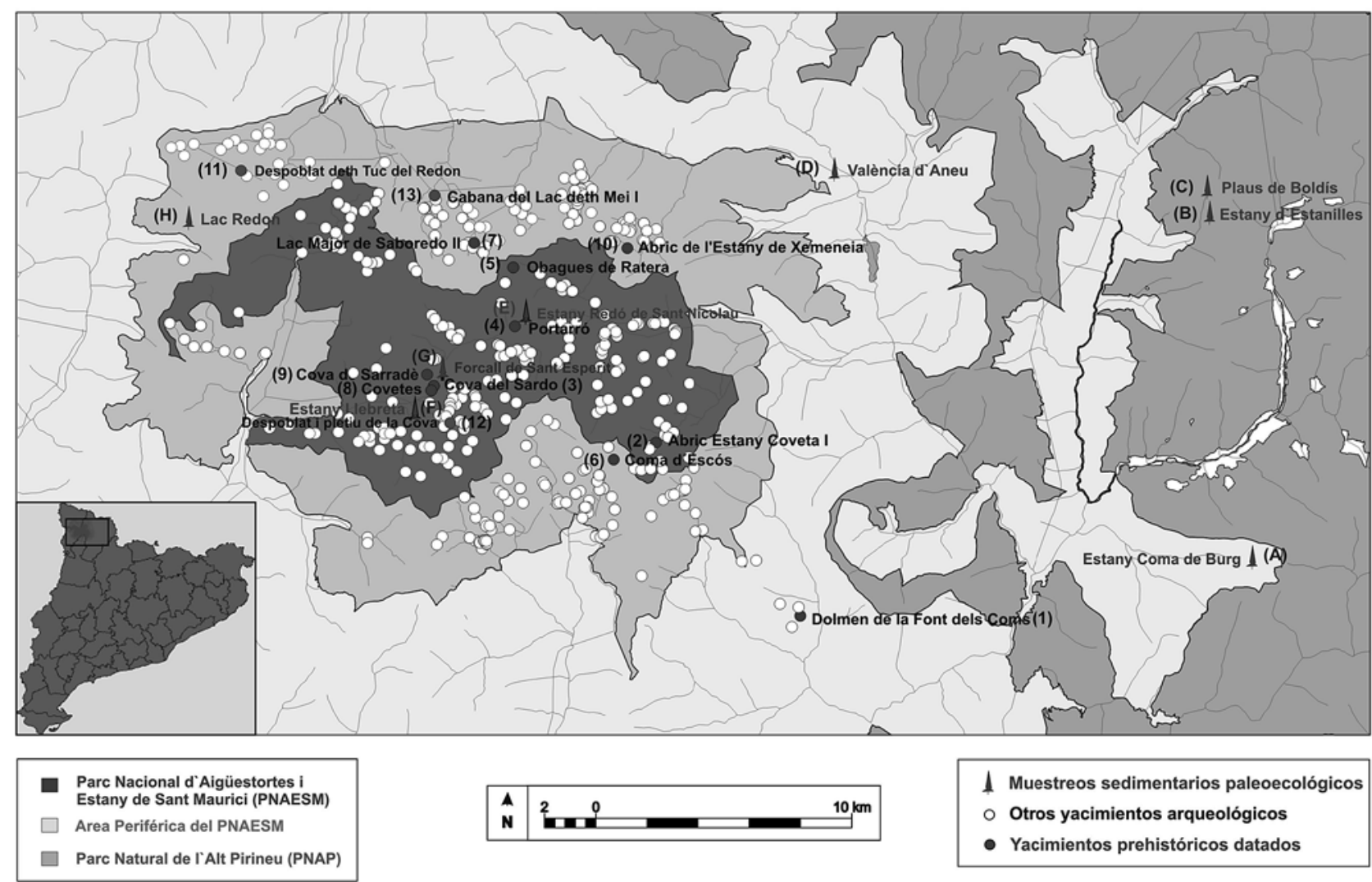

Fig. 1. Mapa topográfico de la zona de estudio, donde se marcan los límites del PNAESM y del PNAP y la hidrografía. Los puntos blancos indican la localización se yacimientos arqueológicos. Los puntos de color gris son yacimientos datados de cronología prehistórica (c. 9000 - 1000 cal ANE): (1) Dolmen Font dels Coms, (2) Abric Estany Coveta I, (3) Cova del Sardo, (4) Portarró, (5) Obagues de Ratera, (6) Coma d'Espòs, (7) Lac Major de Saboredo II, (8) Covetes, (9) Cova de Sarradé, (10) Abric de l'Estany de Xemeneia, (11) Despoblat deth Tuc del Redon, (12) Despoblat i pletiu de la Cova, (13) Cabana deth Lac deth Miei I. Los triángulos indican muestreos paleoecológicos: (A) Estany de la Coma de Burg, (B) Estany d'Estanilles, (C) Plaus de Boldís, (D) València d'Àneu, (E) Estany Redó de Sant Nicolau, (F) Estany Llebreta, (G) Forcall de Sant Esperit, (H) Lac Redon / Topographic chart of the case study area. The boundaries of PNAESM and NPAP and hydrography are marked. The white dots indicate the location of archaeological sites. The gray dots are the sites dating from prehistoric chronology (ca. 9000 - 1000 cal BC): (1) Dolmen Font dels Coms, (2) Abric Estany Coveta I, (3) Cova del Sardo, (4) Portarró, (5) Obagues de Ratera, (6) Coma d'Espòs, (7) Lac Major de Saboredo II, (8) Covetes, (9) Cova de Sarradé, (10) Abric de l'Estany de Xemeneia, (11) Despoblat deth Tuc del Redon, (12) Despoblat i pletiu de la Cova, (13) Cabana deth Lac deth Miei I. Los triángulos indican muestreos paleoecológicos: (A) Estany de la Coma de Burg, (B) Estany d'Estanilles, (C) Plaus de Boldís, (D) València d'Ȧneu, (E) Estany Redó de Sant Nicolau, (F) Estany Llebreta, (G) Forcall de Sant Esperit, (H) Lac Redon..

\section{3.- RESULTADOS}

\subsection{El medio natural: cambios climáticos e im- pacto antrópico}

Desde que finaliza el último episodio frío del Tardiglacial, hacia el 9500 cal ANE, se establece un clima marcadamente estacional en la región pirenaica, caracterizado por un elevado grado de continentalidad, durante la primera mitad del Holoceno (del 9500 al 3200 cal ANE). Éste se define por unas temperaturas de verano más elevadas que las actuales y unas invernales algo más bajas. Ésta dinámica alcanza su cénit durante el óptimo climático (entre 5500 y 3200 cal ANE), cuando tiene lugar el periodo más cálido y húmedo del Holoceno, caracterizado en la zona de estudio por unas temperaturas medias del periodo verano-otoño de hasta 4 $\mathrm{C}^{\circ}$ más elevadas que las actuales (Figura 2). Entre el $3200 \mathrm{cal}$ ANE y $700 \mathrm{cal}$ ANE se desarrolla un periodo de progresiva reducción de la continentalidad y de las tem- peraturas en el verano. Durante los últimos 2700 años se desarrolla el clima actual, que, no obstante, presenta un periodo denominado "Pequeña Edad del Hielo", que dura entre el 1300 y el 1850 cal NE, y que en nuestra zona de estudio se caracteriza por unos inviernos algo más fríos que los actuales, que posibilitaron el desarrollo de pequeños glaciares rocosos en los circos más elevados (BORDONAU 1992, CATALAN et al. 2013).

A partir del análisis de los diversos proxy ambientales (polen, micro-algas, micro-carbones, materia orgánica, metales pesados, etc.) estudiados en la zona, se podrían definir tres grandes periodos en la secuencia de los últimos 12 mil años en la evolución del paisaje pirenaico: 1) Un primer periodo (entre 9500 y $5500 \mathrm{cal}$ ANE) que comprende el la primera mitad del Holoceno, en el cual los cambios en la vegetación vienen condicionados principalmente por el clima, que sufre un proceso de paulatino calentamiento, en un contexto climático marcadamente continental (PĖLACH et al. 2012). 2) Un 


\section{Anomalía térmica $\left(\mathrm{C}^{\circ}\right)$}

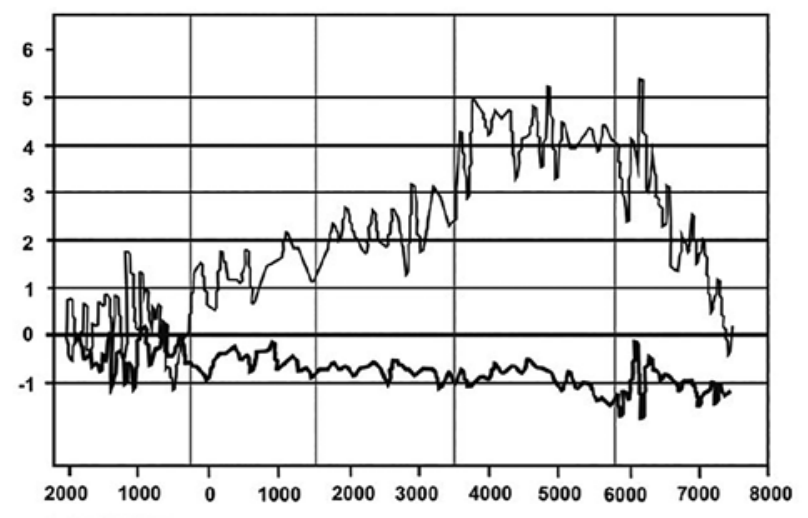

Cal ANE/NE segundo periodo, que se sitúa en torno al Holoceno medio y final (entre 5500 y 700 cal ANE) y que se caracteriza por un paisaje modulado tanto por el clima como por un impacto humano variable y de intensidad creciente (GALOP 2006, GALOP et al. 2007, PÈLACHS et al. 2007). Este periodo comprende desde el Óptimo climático del Holoceno (entre 5500 y 3200 cal ANE), mucho más estable, cálido y húmedo que en el periodo anterior, hasta el Subboreal (entre 3200 y 700 cal ANE) progresivamente más frío e inestable (PÈLACH et al. 2011), sobre todo en cuanto a la disminución de la temperatura y duración de los veranos (CATALAN et al. 2013). 3) Un tercer periodo que comprende el Holoceno más reciente, los últimos 2700 años, que podrimos denominar informalmente antropoceno (GIBBARD y WALWER 2014), y que se caracteriza por la configuración de un paisaje altamente antropizado (PÈLACHS et al. 2007), dominado por una gestión intensiva de las zonas de pastos de altura (MAZIER et al. 2009) y por el desarrollo de zonas de cultivo elevadas en terrazas hasta los 2200 m (BAL et al. 2010). El área del PNAESM vive su momento de máxima presión social durante la plena Edad Media (entre el 1000 y el 1450 cal NE), como demuestran tanto los indicadores polínicos y sedimentarios (PÈREZ-OBIOL et al. 2012) como los datos arqueológicos (RENDU 2003, GARCIA-CASAS el al. 2013) (Figura 3).

\subsection{Distribución espacial de la ocupación}

De la distribución espacial de los asentamientos podemos inferir algunas tendencias. La primera es que, efectivamente, la mayor parte de las estructuras documentadas podrían corresponder a construcciones

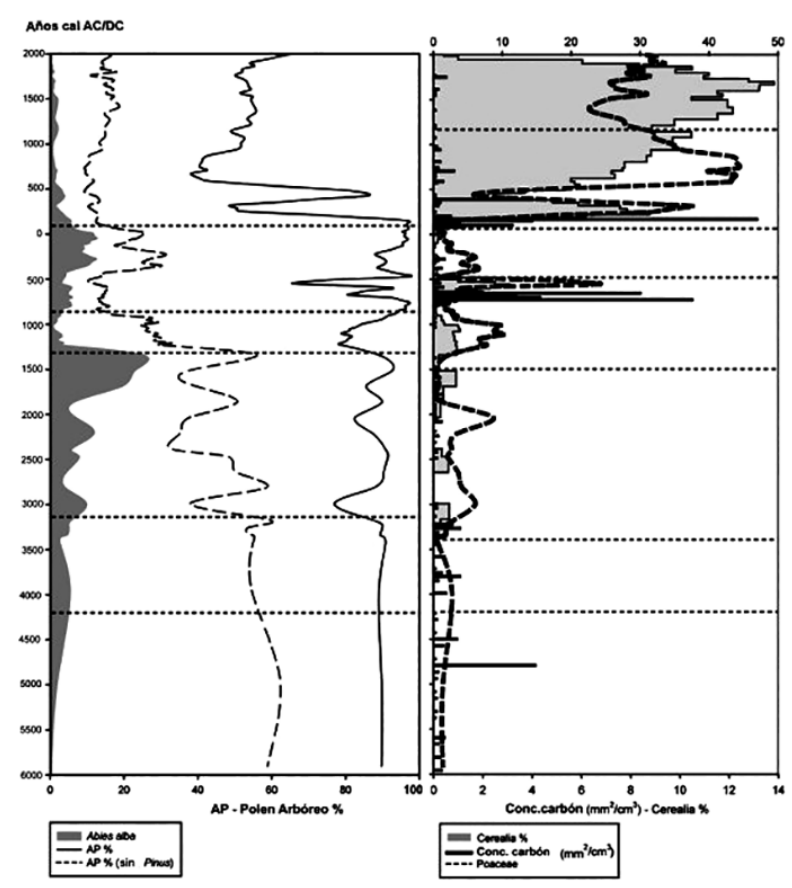

Datos obtenidos de las columnas sedimentarias del Estany de la Coma de Burg (PÈLACHS et al. 2007, 2011 y 2012).
700 cal ANE - Presente

- Elevada y constante presión humana en el medio. Fuere deforestación a todas las cotas Paisaje abierto. Mantenimiento de zonas de pastos mediante el uso de incendios. Cultivo en terrazas. Valores mínimos de bosque máximos de Cerealia y Poaceae.

\section{0 - 700 cal ANE}

- Impacto antrópico moderado y desigual. Uso del fuego para la apertura del paisaje forestal. Presencia de zonas de cultivo en fondos de valle y primeras fondos de valle y primeras
terrazas. Primeros indicadores de ganaderia (Rumex, Polygonum, Plantago) y de agricultura (Cerealia)

5500 - 3300 cal ANE

- Primeros signos de impacto humano puntual en el paisaje. Señales recurrentes de incendios, también en fondos de valle. Indicadores arqueológicos de ocupación sobre los 1500 - 1800 $\mathrm{m}$.
Fig. 3. La figura muestra dos gráficas extraídas de las columnas sedimentarias del Estany de la Coma de Burg (Vall de Cardós, Lleida). A la izquierda se muestran las variaciones en la concentración de polen de abeto (Abies alba) y de polen arbóreo total. A la derecha la variación en la concentración de carbones procedentes de eventos de incendio y el polen de cereal / The image shows two graphs extracted from the sedimentary cores of Estany de la Coma de Burg (Vall de Cardós, Lleida). On the left, are the variations in the concentration of fir pollen (Abies alba) and total arboreal pollen. On the right, the graph shows the variation in the concentration of charcoals from fire events and cereal pollen. 
asociadas con la gestión ganadera de cronología histórica (GARCIA-CASAS 2013). Si vemos el porcentaje de yacimientos documentados, el $63 \%$ se localizan en zonas de pradera alpina, que suponen algo menos del $30 \%$ de la superficie total del terreno de área de estudio y generalmente se sitúan entre 2000 y 2500 m s.n.m., (GASSIOT et al. 2013). Si nos ceñimos a los yacimientos prehistóricos, el porcentaje es diferente, ya que de 12 yacimientos prehistóricos datados entre 9000 y $1000 \mathrm{cal}$ ANE (Tabla 1), sólo 4 son al aire libre. De éstas, las 3 que presentan arquitectura: Coma d'Espós, Despoblat i pletiu de la Cova y Despoblat del Tuc deth Lac Redon, se sitúan al inicio de las zonas de pasto, entre 2120 y 2411 m s.n.m. Las 3 cuevas, o abrigos bajo cornisas, se localizan en paredes rocosas cercanas al fondo del valle de Sant Nicolau, entre 1790 y 1910 m s.n.m.: Cova del Sardo, Cova de Serradé y Covetes. Los restantes 5 abrigos rocosos, formados por bloques erráticos en zonas de circo, se sitúan en las zonas más elevadas, entre 2280 y 2460 m s.n.m.: Portarró, Obagues de Ratera, Lac major de Saboredo II, Abric de l'Estany de Xemeneia y Abric de l'Estany de la Coveta I. Podemos asumir que seguramente hay un cierto sesgo en la localización de estructuras al aire libre de cronologías prehistóricas, a favor de las ocupaciones de abrigos, ya que a priori presentan mejores condiciones para la preservación de los niveles prehistóricos que las construcciones al aire libre.

\subsection{Secuencia de la ocupación}

\subsubsection{La frecuentación mesolítica (c. $9000-5500$ cal ANE)}

Del estudio de los 55 yacimientos de los que disponemos de dataciones radiocarbónicas, podemos inferir que la presencia humana en las zonas de alta montaña del Pirineo axial central se remota al inicio del Holoceno, tras la retirada de los glaciares de valle, dado que la primera ocupación documentada en la zona se ha datado en 8746-8563 cal ANE. Se emplaza aproximadamente un milenio después del último evento glaciar, el Dryas reciente, que en fechas calibradas situamos entre el 10700 y el 9750 cal ANE (PÈLACHS et al. 2012). Se trata de una ocupación al aire libre, documentada bajo el túmulo del Dolmen de la Font del Coms, que se sitúa al SE del parque, a 1840 m s.n.m., en la cabecera del valle de Baiasca. Se trata de una única fase de ocupación asociada a un agujero de poste y con presencia de industria lítica en sílex alóctono (GASSIOT et al. 2013).

La segunda ocupación documentada en la zona de estudio es del Abric de l'Estany de la Coveta I. Es un pequeño abrigo rocoso, de unos $5 \mathrm{~m}^{2}$, situado a $2456 \mathrm{~m}$ de altitud, en el circo glaciar de Peguera. Este sitio presenta una primera ocupación datada en 6830-6589 cal ANE, de la cual se infiere un uso efímero del abrigo, orientado a la gestión de productos cinegéticos, como se deduce del análisis funcional de la industria lítica (GASSIOT et al. 2013 y GARCIA et al. 2008).

La última ocupación datada del periodo se produce en la Cova del Sardo, una cornisa de unos $25 \mathrm{~m}^{2}$, a la que se suma una terraza de unos $50 \mathrm{~m}^{2}$, en su zona exterior. Se ubica a $80 \mathrm{~m}$ sobre río que marca el fondo del valle de Sant Nicolau, a 1790 m s.n.m., orientada al sur, en solana. La primera ocupación de la cornisa data del 5609-5376 cal ANE, y parece tratarse también de una ocupación de baja intensidad, sin presencia de material cerámico. Se asocia a una pequeña cubeta de combustión bajo la cornisa (GASSIOT et al. 2014).

La cronología de estos primeros indicios de presencia humana en el área coincide con la de los vestigios más antiguos documentados en el Valle del Madriu, en Andorra (ORENGO et al. 2014) y plantea la presencia de grupos de cazadores-recolectores explotando al menos puntualmente los medios alpinos y subalpinos al inicio del Holoceno (MARTLUFFet al. 2012).

\subsubsection{La primera ocupación neolítica (c. 5200-4300 cal ANE)}

Es a partir del $\mathrm{V}$ milenio cal ANE, cuando se reconoce la ocupación más intensiva de la Cova del Sardo, con la construcción de un pequeño aterrazamiento exterior y el uso de varios hogares bien estructurados (GASSIOT et al. 2010 y GASSIOT et al. 2014: 269). Esta fase de ocupación (N.8) ha sido datada con 6 fechados radiocarbónicos entre 4802 y 4368 cal ANE (Tabla 1). Podría ser además inmediatamente posterior a un primer evento de incendio documentado en un perfil de suelo, datado en 5200-4940 cal ANE, que podría implicar una apertura intencional de claros para la creación de zonas de pasto y, eventualmente de cultivo, análoga a las que han sido estudiadas en otras regiones de los Pirineos en cronologías similares (BAL et al. 2010, RIUS et al. 2009, PÉREZ-DÍAZ et al. 2015). Los estudios polínicos y arqueobotánicos realizados en los depósitos sedimentarios del yacimiento y de su entorno (OBEA et al. 2011), parecen apuntar en idéntica dirección, al observarse un "aclaramiento" del bosque mixto dominante en la zona y un aumento de la extensión de zonas de pradera, coincidente con esta fase de ocupación del sitio (GASSIOT et al. 2012). Esta hipótesis es coherente con una cierta actividad pastoral estacional y el mantenimiento de zonas deforestadas en el entorno del fondo del valle de Sant Nicolau, dando lugar a un paisaje donde conviviría el bosque con las zonas de pasto mantenidas mediante el uso del fuego.

\subsubsection{La estabilización de la ocupación neolítica (c. 4200- 3500 cal ANE)}

Posteriormente la Cova del Sardo continúa siendo ocupada de manera ocasional, sin grandes modificaciones. Entre 4229 y 3375 cal ANE se han obtenido otros 6 fechados radiocarbónicos en diversas ocupaciones sucesivas del interior de la cornisa del abrigo, asociadas a hogares dispersos. Estas ocupaciones, a nivel arqueológico se asocian con el mantenimiento de industria lítica y el uso de cerámica, así como con el consumo de fauna doméstica (ovicápridos) (GASSIOT et al. 2015). Un se- 
gundo abrigo situado bajo otra cornisa, $400 \mathrm{~m}$ al oeste, a 1880 m s.n.m., el Abric de Covetes, parece tener al menos una ocupación contemporánea a esta fase, que no ha sido datada. Este periodo, a nivel paleoambiental, parece asociarse con la recuperación del entorno forestal del fondo del valle, ahora dominado por el pinar (GASSIOT et al. 2012).

\subsubsection{La expansión de la ocupación en el neolítico final (c. 3500-2300 cal ANE)}

Es en el periodo inmediatamente posterior, entre el 3484 y el 2345 cal ANE cuando se observa un cambio de escala en la gestión y ocupación de la zona de estudio, al haberse documentado un mínimo de 10 yacimientos, mediante 12 fechados distintos (Tabla 1), en este intervalo, la mayoría en pequeños abrigos bajo bloques o cornisas. Además se documenta, al menos, una construcción al aire libre: Coma d'Espós, situada a 2230 m s.n.m., en la Vall Fosca, y una ocupación también al aire libre en el Despoblat del Tuc deth Lac Redon, situado a 2441 m s.n.m., en el Valle de Aran. De éstos sitios, 4: Cova del Sardo, Cova del Sardo II, Covetes y Cova de Sarradé, se ubican entre 1790 y 1910 m s.n.m., cerca del fondo del valle de Sant Nicolau de Aigüestortes, en zonas de bosque mixto. Mientras, los 4 abrigos restantes: Abric de l'Estany de la Coveta I, Portarró, Obagues de Ratera y Lac major de Saboredo II se localizan en zonas elevadas, próximos a cursos de agua, entre 2283 y 2456 m s.n.m., en áreas actualmente poco forestadas o de pasto. Aunque los estudios arqueobotánicos y polínicos indican una mayor extensión forestal para aquel periodo y un límite superior del bosque más elevado que los actuales (CUNILL at al. 2012). Sin embargo, justamente esta primera ocupación de las zonas de mayor altura del piso alpino es coincidente con las primeras evidencias de impacto antrópico, deforestación e incendio en este piso altitudinal (RIUS et al. 2012, GALOP 2006). También es en este período posterior al 3500 cal ANE cuando se constata en otras zonas de la cordillera un claro incremento tanto de las evidencias arqueológicas como del impacto antrópico en el paisaje; como en el Valle del Madriu, en Andorra (ORENGO et al. 2014), o en la Montanya d'Enveig, en el norte de la Cerdanya (RENDU 2003, BAL et al. 2010).

\subsubsection{El cambio en el modelo de gestión en el final de la prehistoria (c. 2200-300 cal ANE)}

Sorprendentemente, y en contra de lo que sugieren los indicadores polínicos y paleoambientales, que indican un creciente impacto antrópico en el medio (PÈLACHS et al. 2007), el periodo siguiente viene marcado por una escasa presencia de evidencias arqueológicas. En conjunto, sólo disponemos para la zona de estudio de dos dataciones radiocarbónicas de yacimientos del II milenio cal ANE: el Abric de l'Estany de Xemeneia, situado en un circo glaciar del Valle de Aran a 2429 m s.n.m., y el Despoblat i pletiu de la Cova, situado en Vall de Llacs a 2121 m s.n.m., en una vía de paso a los pastos de mayor altura. El primer sitio se trata de un pequeño abrigo rocoso con una fase de ocupación datada en 16821464 cal ANE. El segundo sitio se trata de un verdadero poblado de época medieval, datado entre el 1000 y el 1450 cal NE (GARCIA-CASAS et al. 2013). No obstante, a partir tanto del estudio de material cerámico como de la industria de talla laminar en sílex, se ha podido definir una fase prehistórica de ocupación de este yacimiento, de la Edad del Bronce, datada en 1310-1120 cal ANE. Asimismo, se documentan un total de 5 depósitos de cerámica en cavidades rocosas, que podríamos adscribir, por su tipología, a la Edad del Bronce (GASSIOT et al. 2010 y 2014).

En este periodo también situamos una serie de construcciones megalíticas documentadas principalmente al sur de la zona de estudio, como son el Dolmen de la Cabaneta d'Envall situado a 1327 m s.n.m. en la Serra de la Cabaneta, o el Dolmen de la Font del Coms, excavado en su totalidad y que se sitúa también en una zona elevada de la cabecera de la Vall de Baiasca, a 1850 m n.s.m. (GASSIOT et al. 2014). Asimismo, destaca la presencia de construcciones de planta circular: Túmul de la Font de la Portella, Túmul de la Pleta d'Erdo y Túmul de les Cometes de Casesnoves, situadas entre 2200 y 2300 m s.n.m. (GARCIA CASAS et al. 2014). Tipológicamente parecen corresponder con las de la primera Edad del Hierro, si tomamos como referencia otras muy similares fechadas al norte de la zona de estudio, en el Pla de Beret (Valle de Aran) y que datan de principios del I milenio cal ANE (LOPEZ y PONS 1995).

\subsubsection{La época histórica}

Ya en periodos históricos, a partir del s. II cal ANE, y más aún en época visigótica, a partir del s. V y altomedieval, a partir del s. IX, se documentan diversos picos de presión antrópica en la zona de estudio, que alcanza su cénit en torno al siglo XIII (PÈLACH et al. 2007), cuando la densidad de ocupación en la Vall de Boí es la máxima histórica, que coincide con el auge del feudalismo en la región. Es en este periodo cuando se establecen verdaderos poblados (posiblemente estacionales) en zonas de altura, sobre los 2000 m s.n.m. (GARCIA-CASAS et al. 2013, GASSIOT et al. 2013).

\section{4.- DISCUSIÓN: LA CONSTRUCCIÓN DEL PAI- SAJE PASTORAL}

Gracias al desarrollo de este enfoque interdisciplinar se puede explicar el paisaje como la consecuencia de al menos cuatro vectores: clima, orografía, dinámica de la vegetación e impacto antrópico, que interactúan a lo largo del tiempo, co-evolucionando (FOLKE 2006), y generando un paisaje que también fue profundamente social (REDMAN et al. 2004). En el caso de estudio que aquí analizamos, las zonas de alta montaña del Pirineo, coincide además que el ecosistema resultante de la actuación humana 
en el medio, a lo largo de los últimos 7000 años, da como resultado un territorio esencialmente pastoral; a pesar de que las actividades productivas que se vienen realizando en las zonas de alta montaña desde la prehistoria (caza, recolección, pesca, obtención de maderas, minería, metalurgia, agricultura, etc.) sean múltiples y en muchos casos complementarias. Sin duda, de todas, la actividad que más ha impactado en el modelado del actual medio ambiente es la ganadería estacional, móvil o trashumante (RENDU 2003, GARCIA CASAS 2013, ROJO GUERRA et al. 2013, CLEMENTE et al. 2014, MAZZUCCO 2014).

A partir del estudio de los archivos sedimentarios, ya comienza a intuirse un primer impacto en el medio natural a mediados del Holoceno, entre el 5500 y el 4500 cal ANE (GALOP 2006, PĖLACHS et al. 2007, RIUS et al. 2012). Si nos fijamos en los datos de impacto ambiental más directamente relacionados con las ocupaciones arqueológicas, vemos que efectivamente, la escasa incidencia en la vegetación que se observa en términos globales para este periodo (5500-3500 cal ANE) (CATALAN et al. 2001), se ve claramente magnificada en los datos polínicos de los depósitos arqueológicos. En el caso de la Cova del Sardo, la máxima incidencia humana en el paisaje del entorno del abrigo, en los 3 milenios de su larga secuencia prehistórica (5609-2495 cal ANE), se sitúa a mediados del V milenio, entre el 4802 y el 4368 cal ANE, cuando se detecta una clarísima apertura del bosque y un mantenimiento de zonas de pasto, como indican el marcado descenso del polen arbóreo y el aumento de las herbáceas (Poaceae, Asteraceae y Plantago) (GASSIOT et al. 2012). A tenor de los datos preliminares del perfil del Forcall de Sant Esperit situado a $1771 \mathrm{~m}$ s.n.m., a $570 \mathrm{~m}$ al noreste de la Cova del Sardo, se define un evento importante de incendio del fondo del valle que data de 5200-4940 Cal ANE, apenas uno o dos siglos anterior a la primera fase de ocupación intensiva de la Cova del Sardo. De manera que parece probable que la ocupación del valle de Sant Nicolau fuera precedida por una apertura del bosque usando el fuego, justo a inicios de la ocupación neolítica del Pirineo central. En otras zonas más elevadas, como en la Turbera de Estanillas, en la falda del Montarenyo, a 2247 m s.n.m., también se detectan las primeras fases de incendio en torno al 5500 cal ANE y la presencia de indicadores polínicos de áreas de pasto (Artemisia, Plantago, Rumex) en torno al 5000 cal ANE (PÈREZ OBIOL et al. 2012; CUNILL et al. 2012). Un poco más al sur, en la vertiente norte del Turbón, se observa también la creación de pastizales al final del VI Milenio, precediendo la fase de ocupación más antigua de la Cueva de Els Trocs, entorno a los 1500 m s.n.m. (URÍA BLANCO 2013).

La posibilidad de un primer desarrollo de campos de cultivo en este periodo del neolítico antiguo ( $\mathrm{V}$ milenio cal ANE), que efectivamente han sido documentadas en otros valles inferiores de la región (GALOP et al. 2003), también en el valle de Sant Nicolau, no es incompatible con las condiciones ambientales del periodo, el más cálido del Holoceno, marcado por ciclos de verano-otoño sustancialmente más cálidos que los actuales, según indican los datos polínicos (CATALAN et al. 2001) y el estudio de diatomeas y crisofíceas en la zona (PLA y CATALAN 2005, CATALAN et al. 2013). No obstante, en el momento actual de la investigación carecemos de datos suficientes que certifiquen esta posibilidad, ya que la presencia puntual de restos carpológicos de cebada (Hordeum vulgare var. nudum), en una estructura de combustión de la Cova del Sardo, datada en 4681-4462 cal ANE (GASSIOT et al. 2012), así como algunos restos fitolitológicos y polínicos de posibles cereales domésticos, aún en fase de estudio (CATALAN et al. 2013), dejan esta línea de investigación aún abierta.

Esta dinámica ambiental se ve claramente alterada en el III milenio cal ANE, que se caracteriza por un marcado cambio climático en toda la Europa mediterránea y que en la región pirenaica se concreta en un aumento de la aridez y en un descenso de las temperaturas en verano. Lo que explica el desarrollo de bosques de coníferas a menor altitud, y la expansión del abeto (Abies alba) y del haya (Fagus sylvatica) en zonas de alta montaña, que van sustituyendo los bosques mixtos de caducifolias (Quercus, Betula, Corylus), antes dominantes en los fondos de valle hasta los 2000 m s.n.m. (PĖLACHS et al. 2007). Paralelamente, se detecta un incremento en el índice de polen de cereales domésticos (PÈLACHS et al. 2007) y un aumento de la extensión de las praderas alpinas (CUNILL et al. 2012). Climáticamente este periodo coincide con una disminución de la depositación de materia orgánica en los lagos (PÈLACHS et al. 2011) que se asocia al Bond event 3, y que se ha asociado también a un descenso de la temperatura del verano-otoño (CATALAN et al. 2013). Estos indicadores además coinciden con una disminución del polen arbóreo (CATALAN et al. 2001, PÈLACHS et al. 2007). Todos estos cambios en el paleopaisaje de la zona de estudio hacen pensar en la interacción de factores climáticos y dinámicas sociales de intensificación en la explotación de las zonas de alta montaña, como también sugiere el registro arqueológico.

\section{CONCLUSIONES}

Si tomamos el caso del valle de la Valira, en Andorra, como ejemplo, se detecta una primera frecuentación humana de las zonas de alta montaña, en la cabecera del Madriu, inmediatamente posterior a la retirada de los glaciares (ORENGO et al. 2014), similar a la que observamos en nuestra zona de estudio. Éstas ocupación estaría asociada a sociedades cazadoras-recolectoras que practicarían economías extractivas de tipo mesolítico, cuya continuidad durante toda la primera mitad del Holoceno, desde el X al VII milenio cal ANE, se documenta en el abrigo de la Balma Margineda, situada a 950 m s.n.m., en el fondo del Valle de la Valira (GUILAINE el al. 1985, MARTZLUFF et al. 2012). Los datos paleoambientales no indican elementos de antropización del paisaje para este periodo en la zona del 
PNAESM (CATALAN et al. 2001), aunque los picos de incendio son frecuentes a inicios del Holoceno en otras zonas de estudio, como la Vall de Cardós en el PNAP (Pèlachs et al. 2007, CUNILL et al. 2012). Se discute si su causa podría ser antrópica o climática (PÈREZOBIOL et al. 2011).

A tenor de los datos de la distribución arqueológica de las ocupaciones, y en relación con los datos paleoambientales discutidos, se plantea la hipótesis de un primer poblamiento neolítico, basado en la explotación pastoral estacional de las zonas de alta montaña, entre finales del VI e inicios del V milenio cal ANE (ROJO-GUERRA et al. 2013). Ésta ocupación vendría precedida del uso del fuego, como elemento de gestión en la apertura del bosque y posterior mantenimiento de zonas de pasto, y eventualmente también de cultivo, como ha sido documentado en otras áreas de la región pirenaica, tanto al norte (GALOP el al. 2003, RIUS et al. 2009 y 2012) como al sudeste (OMS et al. 2016) o al sudoeste de la cordillera (MAZIER et al. 2009, PÉREZ DíAZ et al. 2015). Ésta primera ocupación neolítica, a diferencia de la frecuentación mesolítica previa, si tendría un impacto limitado en el paisaje, y podría centrarse más en zonas cercanas a los yacimientos, donde se mantendrían abiertas las zonas de pasto; y no tanto de una ocupación del piso alpino, todavía altamente forestado. Así sugieren los resultados del estudio del impacto en la vegetación durante las ocupaciones del neolítico antiguo de la Cova del Sardo (GASSIOT et al 2012) o de la cercana Cova dels Trocs, situada a 1564 m s.n.m. (URíA et al., 2013, LANCELOTTI et al. 2014).

El modelo de ganadería que se propone para el neolítico antiguo, a tenor de los análisis de materiales de la Cova del Sardo (MAZZUCCO et al. 2013) y de la Cova dels Trocs (ROJO-GUERRA et al. 2013), sería algún sistema de movilidad estacional altitudinal, desde los valles hasta las áreas de alta montaña, y longitudinal, sur-norte. Se discute si este tipo de patrones de movilidad a larga distancia podrían conectar las poblaciones del alto Pirineo con las del Prepirineo o incluso hasta con las del Valle del Ebro, como apunta el estudio de las materias primas líticas (MAZZUCCO et al. 2013), y si ya se llevarían a cabo prácticas ganaderas de largo recorrido desde el V milenio cal ANE (MAZZUCCO 2014). Se discute si estás prácticas socio-económicas, una vez establecido un sistema pastoral en circuitos de movilidad estacional, longitudinal y altitudinal, se mantendrían a lo largo del tiempo (ROJO-GUERRA et al. 2013, CLEMENTE et al. 2014).

A tenor de los datos arqueológicos presentados en la zona de estudio del PNAESM, parece que el modelo de gestión del territorio cambia progresivamente, desde el $V$ milenio cal ANE. Se intensifica claramente en el III milenio cal ANE, cuando se ocupan por vez primera durante el Neolítico y de manera generalizada las zonas más elevadas, sin abandonarse los refugios del fondo del valle (GASSIOT et al. 2010). Patrón global en toda la cordillera pirenaica que supone un incremento del número de yacimientos datados (GALOP et al. 2007, GALOP 2006, RENDU 2003, ORENGO et al. 2014, MAZZUCCO 2014, GASSIOT et al. 2014) y paralelamente, de la presión antrópica en las zonas alpinas y un aumento de la extensión de las zonas de pasto, junto con una disminución de la superficie forestal total disponible (PÈLACHS et al. 2007, PÈREZ-OBIOL et al. 2012).

Durante el II y I milenio cal ANE, como ya hemos planteado, los indicadores sedimentarios, y particularmente polínicos, sugieren un cierto aumento en la frecuencia de incendios antrópicos que permitiría un mantenimiento y ampliación de las zonas de pasto de altura abiertas ya en el III milenio (PÈLACHS et al. 2007, GALOP et al. 2007, GASSIOT et al. 2014). Por el contrario, si bien la ocupación humana de las zonas de alta montaña no puede ser cuestionada, ante la evidencia de monumentos funerarios (Dólmenes, Crómlech) (LOPEZ y PONS 1995), la presencia de depósitos de cerámica y de ocupación de sitios en abrigo o al aire libre, también debemos admitir que observamos, sin duda, un cambio en el patrón de asentamiento, al abandonarse los pequeños abrigos y cuevas anteriormente utilizados durante el neolítico (GASSIOT et al. 2010, GARCIA CASAS et al. 2014).

En conclusión, éste tipo de dinámicas socio-históricas, que pueden conllevar el desarrollo de prácticas de uso y gestión del medio y de los recursos, como la ganadería de movilidad o trashumante, son cambiantes a lo largo del tiempo. Lo cual exige una visión diacrónica del proceso de ocupación y gestión del territorio. Los paisajes socio-ecológicos, por tanto, cambian o co-evolucionan, debido, tanto al desarrollo de las prácticas productivas de explotación del medio, como a la adaptación, o respuesta, de tales prácticas socio-económicas al propio paisaje existente y a los cambios climáticos. Este tipo de problemáticas procesuales e interacciones entre los sistemas económicos y el cambio ambiental ha sido especial objeto de estudio desde la ecología social (REDMAN et al. 2004, FOLKE 2006). Actualmente, se discute si este tipo de sistemas productivos y dinámicas de gestión del medio ambiente, como la trashumancia son o no resilientes a lo largo del tiempo (MAZZUCO en prensa, ROJO-GUERRRA et al. 2013). Los datos que aquí se discuten parecen apuntar más al cambio, que no a la permanencia, en tales dinámicas sociales.

\section{AGRADECIMIENTOS}

Este programa de investigación ha sido posible gracias a la conjunción de diversas líneas científicas (clima, paleogeografía, arqueología) en un enfoque interdisciplinar integrado; lo cual ha favorecido la continuidad del programa mediante el desarrollo de una serie de proyectos científicos financiados de manera consecutiva desde el año 2001 hasta la actualidad: Prospeccions a l'alta muntanya del Pallars Sobirà (2001 y 2002), La vida prehistòrica a l'alta muntanya del Pa- 
Ilars Sobirà: de la cacera a la transumància (9000-50cal ANE) (de 2003 a 2005), Arqueologia de l'Alta muntanya Pirinenca. Ocupació Humana i canvi climàtic al llarg de l'Holocè (años 2006 a 2009), Interacción entre clima y ocupación humana en la configuración del paisaje vegetal del Parque Nacional de Aigüestortes i Estany de Sant Maurici a lo largo de los últimos 15.000 años (OCUPA) (2010 a 2012); Dinàmiques dels Espais Pirinencs d'Altitud. Un SIG del patrimoni arqueològic i la modelització dels territoris pastorals (DEPART) (2014 y 2015), Análisis ecológico de la culturización del paisaje de alta montaña desde el Neolítico: los Parques Nacionales de montaña como modelo (CUL-PA) (de 2014 a 2016) y Cims, prats, ramats i persones. L'explotació de l'alta muntanya del Parc Nacional d'Aigüestortes i Estany de Sant Maurici al llarg de l'Holocè: una aproximació arqueoecològica (CPRP) (vigente desde 2014 hasta 2017) y Modelizacion de los espacios prehistoricos de montaña. Un sig del patrimonio arqueologico y los territorios pastoriles (vigente de 2016 a 2018).

\section{BIBLIOGRAFÍA}

AUGÉ, O., GASSIOT, E. y PÈLACHS, A.

2012 La producció de ferro a la protohistòria i època romana al Pallars Sobirà. L'exemple del Bosc de Virós a la Vall Ferrera, en VVAA, Boscos de Ferro. Actes de les Primeres Jornades de Recerca i Desenvolupament de la Vall Ferrera. Grasineu Edicions. Tremp: 9-40.

BAL, M.C.; RENDU, C.; RUAS, M.P.; CAMPMAJO, P.,

2010 Paleosol charcoal: reconstructing vegetation history in relation to agro-pastoral activities since the Neolithic. A case of study in the Eastern French Pyrenees, Journal of Archeological Science 37: 1785-1797.

BORDONAU, J.

1992 Els complexos glacio-lacustres relacionats amb el darrer cicle glacial als Pirineus, Geoforma Ed., Logroño.

CAÑELLAS-BOLTÀ, N.; RULL, V.; VIGO, J.; MERCADÉ, A.

2009 Modern pollen-vegetation relationship along an altitudinal transect in the Pyrenees (southwestern Europe), en The Holocene 19, 8 (2009): 1185-1200.

CARNELLI, A.,

2002 Long Term dynamics of the vegetation at the subalpine-alpine ecocline during the Holocene: comparative study in the Alestsch region, Val d'Arpette, and Furka Pass (Valais, Swietzerland), Section des Sciences de la Terra, Universitè de Genève, Suiza.

CATALÁN, J.; PÉREZ-OBIOL, R.; PLA, S.

2001 Canvis climàtics a Aigüestortes durant els darrers 15.000 anys", en V Jornades sobre Recerca al Parc Nacional d'Aigüestortes i Estany de Sant Maurici (2000), Lleida, Departament de Medi Ambient: 45-51.
CATALAN J PÈLACHS, A GASSIOT E ANTOLÍN, F., BALLESTEROS, A., BATALLA, M., BURJACHS, F., BUCHACA, T., CAMARERO, L.' CLEMENTE I. CLOP X GARCÍA D GIRALT' S., JORDANA-LLUCH, L., MADELLA, M., MAZZUCO, N., MUR, E., NINYEROLA, M., OBEA, L., OLTRA, J., PÉREZ-OBIOL, R., PIQUÉ, R., PLA, S., RIVERA-RÓNDÓN, C., RODRÍGUEZ, J.M., RODRÍGUEZ-ANTÓN, D., SÁEZ, A., y SORIANO, J.M.,

2013 Interacción entre clima y ocupación humana en la configuración del paisaje vegetal del Parque Nacional de Aigüestortes i Estany de Sant Maurici a lo largo de los últimos 15.000 años, en RAMÍREZ, L., Y ASENSIO, B. (eds.), Proyectos de investigación en Parques Nacionales: 2009-2012 - Naturaleza y Parques Nacionales. Madrid, Organismo Autónomo de Parques Nacionales: 71-92.

CLEMENTE, I, GASSIOT, E., REY, J. (eds.)

2014 El Sobrarbe antes de Sobrarbe: Pinceladas de historia de los Pirineos. Centro de Estudios de Sobrarbe, Zaragoza, $206 \mathrm{p}$.

CUNILL, R.; SORIANO, J.M.; BAL, M.C.; PÈLACHS, A. Y PÉREZ-OBIOL, $R$

2012 Holocene high-altitude vegetation dynamics in the Pyrenees: A pedoanthracology contribution to an interdisciplinary approach, Quaternary International Volume 289 (2013): 60-70.

DOMINGO, M. (Coord.)

2000 Geopirineos. Espacios naturales y geologia pirenaica. Sector central y centrooriental, AEPECT, Girona.

ESTÉBAN, A., (coord.), OLIVER, J.; CÒTS, P.; PÈLACHS, A.; MENDIZÀBAL, E., SORIANO, J.M.; NASARRE, E.; MATAMALA, N.,

2003 La humanización de las altas cuencas de la Garona y las Nogueras (4500 aC - 1955 dC), Madrid, Servicio Nacional de Parques Nacionales, 2003

GALOP, D.; VANNIERE, B. y LOPEZ-SAEZ, J.A.

2003 Des abattis-brûlis néolithiques au système agro-pastoral pyrénéen actuel. Mise en évidence pluridisciplinaire de l'évolution du système agraire dans une vallée du piémont nord-pyrénéen entre le Néolithique ancien et I'Antiquité tardive, en Actes du XII Colloque international d'archéologie de Puigcerda. Pirineus i veïns al $3 r$ Mil.lenni $A C$, Puigcerda: 82-94.

GALOP, D.,

2006 La conquête de la montagne pyrénéenne au Néolithique, en GUILAINE, J. (coord.), 2006, Populations Néolithiques et enivrements, Ed. Errance, Paris: 279-295.

GALOP, D. ; CAROZZA, L. ; MAREMBERT, F. ; BAL, M.C

2007 Activités agropastorales et climat durant l'Âge du Bronze dans les Pyrénées: l'état de la question à la lumière des données environnemental es et archéologiques, en RICHARD, H. ; MAGNY, M. ; MORDANT, C. (eds.) 2007 Environnements et cultures à l'âge du Bronze en Europe occidentale, CTHS, France.

GARCÍA, V

2008 Análisis del material lítico. En GASSIOT, E. y JIMÉNEZ, J. 2008. Excavació arqueológica de l'Abric de l'Estany de la Coveta I. Memoria final de l'intervenció de juny-setembre de 2005, Barcelona, Área de Coneixement i Recerca, Direcció General de Patrimoni: 83-89. 
GARCIA CASAS, D.; GASSIOT, E.; MAZZUCCO, N.; OBEA, L.; RODRÍGUEZ-ANTÓN, D.

2013 Mes de 2.000 anys d'arquitectura ramadera al Parc Nacional d'Aigüestortes i Estany de Sant Maurici, en L'Investigació al Parc Nacional d'Aigüestortes i Estany de Sant Maurici. Jornades sobre Recerca al Parc Nacional d'Aigüestortes i Estany de Sant Maurici Boí (Alta Ribagorça), 17,18 y 19 d’octubre de 2012. Ed. Generalitat de Catalunya: 261-274.

\section{GARCIA CASAS, D.}

2013 Aproximación al poblamiento de las zonas de alta montaña pirenaicas desde la arqueología y la etnografía, SAGVNTVM (P.L.A.V.) 45 (2013): 221-239.

GARCIA CASAS, D.; GASSIOT BALLBÈ, E.; MAZZUCCO, N.; OBEA, L.; PUIG, E.; RODRÍGUEZ ANTÓN, D.

2014 On són els vius? El poblament de l'Alt Pirineu occidental durant el $2 \mathrm{n}$ i $1 \mathrm{r}$ mil.lenni cal ANE. En MERCADAL I FERNÀNDEZ, O. (coord.) 2014 La Transició Bronze Final - 1a Edat del Ferro en els Pirineus i territoris veïns: XV Col/loqui Internacional d'Arqueologia de Puigcerdà. Congrés Nacional d'Arqueologia de Catalunya. Puigcerdà 17,18 i 19 de novembre de 2011. Puigcerdà: Institut d'EstudisCeretans, pp. 153-166.

\section{GASSIOT, E.; RODRÍGUEZ-ANTÓN, D. Y GARCÍA, V.}

2010 El poblament del Parc Natural de Aigüestortes i l'Estany de Sant Maurici durant el neolític. Noves dades arqueológiques i les seves implicacions per a l'estudi de les zones d'alta muntaya, en VIIII Jornades sobre Recerca al Parc Nacional d'Aigüestortes i Estany de Sant Maurici, Lleida, 2009. Ed. Generalitat de Catalunya: $153-164$

GASSIOT, E.; RODRÍGUEZ-ANTÓN, D.; BURJACHS, F.; ANTOLÍN, F.; BALLESTEROS, A.,

2012 Poblamiento, explotación y entorno natural de los estadios alpinos y subalpinos del Pirineo central durante la primera mitad del Holoceno, en Cuaternario y Geomorfología, Vol 26 (3-4): 29-45.

GASSIOT, E.; GARCIA-CASAS, D.; MAZZUCCO, N.; OBEA, L.; OLIVA, M.; RODRÍGUEZ-ANTÓN, D.; BURJACHS, F.; ANTOLÍN, F.

2013 Una historia de llarga durada. L'ocupació humana del Parc Nacional d'Aigüestortes i Estany de San Mauri$\mathrm{ci} i$ àrees properes durant els darrers 10.000 anys, en 2013-Primeres jornades d'Arqueologia i Palentologia del Pirineu i Aran. Ed. Generalitat de Catalunya: 32-38.

GASSIOT E.; RODRÍGUEZ- ANTÓN, D.; PÈLACHS, A.; PÉREZ OBIOL, R.; JULIÀ, R.; BAL, M., C.; MAZZUCCO, N.,

2014 La alta montaña durante la Prehistoria: 10 años de investigación en el Pirineo catalán occidental, Trabajos de Prehistoria $71, N^{\circ}$ 2: 262-282.

GASSIOT, E.; MAZZUCCO, N.; OBEA, L.; TARIFA, N.; ANTOLIIN, F.; CLOP, X.; NAVARRETE, V. y SANNA, M.

2015 La Cova del Sardo de Boí i l'explotació de l'alta muntanya als Pirineus occidentals en època neolítica, Tribuna d'arqueologia 2013-2014: 199-218.
GASSIOT, E., CLEMENTE, I., MAZZUCCO, N., GARCIA, D.; OBEA, L., RODRIIGUEZ ANTÓN, D.

2016 Surface surveying in high mountain areas, is it possible? Some methodological considerations. Quaternary International (2016) vol. 402: 35-45

GIBBARD, P.L., WALKER, M.J.C.

2014 The term 'Anthropocene' in the context of formal geological classification. In A Stratigraphical Basis for the Anthropocene, Geological Society of London, Special Publications, Vol. 395: 29-37.

GUILAINE, J.; MARTZLUFF, M., GEDDES, D.; COULAROU, J.; LE GALL, O.

1985 Postglacial Enviroments, Settlement and Subsistence in the Pyrenees: the Balma Margineda, Andorra, en CLIVE BONSAL (ed.) 1985 Mesolithic in Europe, Edinbourgh: 561-571.

FOLKE, C.

2006 Resilience: The emergence of a perspective for social-ecological systems analyses, Global Environnemental Change 16 (2006): 253-267.

LANCELOTTI, C.; BALBO, A.L.; MADELLA, M.; IRIARTE, E.; ROJO-GUERRA, M.; ROYO, J.I.; TEJEDOR, C.; GARRIDO, R.; GARCÍA, I; ARCUSA, H.; PÉREZ-JORDÀ, G.; PEÑA-CHOCARRO, L.

2014 The missing crop: investigating the use of grasses at Els Trocs, a Neolithic cave site in the Pyrenees (1564 $\mathrm{m}$ asl), Journal of Archaeological Science 42 (2014): 456-466.

\section{LOPEZ, J y PONS, E.}

1995 Les necròpolis d'incineració tumulària de la zona Pirinenca, en BERTRANPETIT, J.; VIVES, E. (ed.), Muntanyes i població: El passat dels Pirineus des d'una perspectiva multidisciplinària. I Simposi dels Pirineus, Andorra la Vella, Centre de Trobada de les Cultures Pirinenques: 107-125.

MARTZLUFF, M.; MARTÍNEZ-MORENO, J.; GUILAINE, J.; MORA, R; CASANOVA, J.

2012 Transformaciones culturales y cambios climáticos en Ios Pirineos catalanes entre el Tardiglaciar y Holoceno antiguo: Aziliense y Sauveterriense en Balma de la Margineday Balma Guilanyà. Cuaternario y Geomorfología, 26 (3-4), 61-78.

MAZIER, F.; GALOP, D.; GAILLARD, M.D.; RENDU, C.; CUGNY, C.; LEGAZ, A.; PEYRÖN, O.; BUTTLER, A.

2009 Multidisciplinary approach to reconstructing local pastoral activities: an example from the Pyrenean Mountains (Pays Basque), en The Holocene 19 (2): 171-188.

MAZZUCCO, N., GASSIOT, E.; ORTEGA, D.; CLEMENTE, I.; RODRIGUEZ ANTÓN, D.

2013 Lithic procurement at the Cova del Sardo during Neolithic: preliminary data on mobility strategies, Archeologia Postmedievale 17 (2013): 87-96.

\section{MAZZUCCO, N.}

2014 The human occupation of the southern central Pyrenees in the sixth-third millennia cal BC: a traceological analysis of flaked stone assemblages, Ph.D Dissertation, Universitat Autònoma de Barcelona, $432 \mathrm{p}$. 
MONTSERRAT, J. M.

1992 Evolución glaciar y postglaciar del clima y la vegetación en la vertiente sur del Pirineo: Estudio Palinológico, Zaragoza, Instituto Pirenaico de Ecología - C.S.I.C.

NINOT, J.M. Y FERRÉ, A.

2008 Plant diversity across five vegetation belts in the Pyrenees (Catalonia, Sapain), en Collectanea Botanica (Barcelona), vol. 27 (2008): 65-74.

OBEA, L.; PIQUÉ, R; MARTIN, M. Y GASSIOT, E.

2011 The exploitation of forest resources in mountain areas during the Neolithic in the northeast of the Iberian Peninsula, en Archaeological charcoal: natural or human impact on the vegetation. Sagvntvn Extra 11: 129-130.

OMS, X., GIBAJA, J.F., MAZZUCCO, N. y GUILAINE, J.

2016 Revisión radiocarbónica y cronocultural del Neolítico Antiguo de la Balma Margineda (Aixovall, Andorra). Trabajos de Prehistoria, 73(1):29-46.

ORENGO, H.A., PALET, J. M., EJARQUE, A., MIRAS, Y., RIERA, S.

2014 Shifting occupation dynamics in the Madriu-Perafita-Claror valleys, Quaternary International 353: 140152.

PÈLACHS, A.; SORIANO, J.M.; NADAL, J.; ESTEBAN, A.

2007 Holocene environmental history and human impact in the Pyrenees. Contributions to Science, 3 (3), 423-431.

PÈLACHS, A.; JULIÀ, R.; PÉREZ-OBIOL, R.; SORIANO, J.M., BAL, M.C.; CUNILL, R. Y CATALAN, J.

2011 Potential influence of Bond events on mid-Holocene climate and vegetation in southern Pyrenees as assessed from Burg lake LOI and pollen records, en The Holocene 21(1): 95-104

PÈLACHS, A.; RODRÍGUEZ, J.M.; PÉREZ-OBIOL, R.; JULIÀ, R.; BURJACHS, F.; EXPÓSITO, I.; CUNILL, R.; SORIANO, J.M. E YLL, R.

2012 Dinámica del clima y del paisaje vegetal del pirineo de Lleida durante la transicion Tardiglaciar-Holoceno, en Cuaternario y Geomorfología 26 (3-4): 79-96.

PÉREZ-DÍAZ, S.; LÓPEZ-SÁEZ, J.A.; GALOP, D.

2015 Vegetation dynamics and human activity in the Western Pyrenean Region during the Holocene, Quaternary International 364: 65-77.

PÉREZ-OBIOL, R.; BAL, M-Cl.; PÈLACHS, A.; CUNILL, R.; SORIANO, J.M

2012 Vegetation dynamics and anthropogenically forced changes in the Estanilles peat bog (southern Pyrenees) during the last seven millennia, Vegetation History and Archaeobotany 21: 385-396

PLA, S.; CATALAN, J.

2005 Chrysophyte cysts from lake sediments reveal the submillennial winter/spring climate variability in the northwestern Mediterranean region throughout the Holocene, Climate Dynamics (2005) 24: 263-278.
REDMAN, CH. L.; FISH, P.R. y JAMES, S.R. (Eds.)

2004 The Archaeology of Global Change: The Impact of Humans on Their Environment, Smithsonian Institute Press.

RENDU, Ch.

2003 La montagne d'Enveig: une estive pyreneen dans la longue durée, Ed.Trabucaire, Perpinyà.

RIUS, D.; VANNIÈRE, B; GALOP, D.,

2009 Fire frequency and landscape management in the northwestern Pyrenean piedmont, France, since the early Neolithic (8000 cal. BP), en The Holocene 19 (6): 847 859.

2012 Holocene history of fire, vegetation and land use from the central Pyrenees (France), en Quaternary Research 77 (2012): 54-64

ROJO-GUERRA M.A.; PEÑA-CHOCARRO, L.; ROYO-GUILLÉN, J.I.; TEJEDOR-RODRÍGUEZ, C.; GARCÍA-MARTÍNEZ DE LAGRÁN, I.; ARCUSA-MAGALLÓN, H.; GARRIDO-PENA, R.; MORENO-GARCÍA, M.; MAZZUCO, N.; GIBAJA-BAO, J.F.; ORTEGA, D.; KROMER, B.; ALT, K.W.

2013 Pastores trashumantes del Neolítico Antiguo en un entorno de alta montaña: secuencia crono-cultural de la Cova de Els Trocs (San Feliú de Veri, Huesca), BSAA arqueología, LXXIX, 2013, Ediciones Universidad de Valladolid: 9-55.

SORIANO, J.M.; ESTEBAN AMAT, A.; PÈLACH, A.

2003 La antropización del paisaje vegetal del Parque Nacional de Aigüestortes i Estany de Sant Maurici a partir de la biogeografía, en: 181-190.

URÍA-BLANCO, N

2013 Registros sedimentarios como indicadores paleoambientales y de la actividad antrópica durante la Neolitización: La Cueva de Els Trocs y su entorno (Abella, Huesca). CKQ Estudios de Cuaternario/Leioa 3, pp: 123-134. 